\title{
Virtual Consultations Through the Veterans Administration SCAN-ECHO Project Improves Survival for Veterans With Liver Disease
}

\author{
Grace L. Su, ${ }^{1,2}$ Lisa Glass, ${ }^{1,2}$ Elliot B. Tapper, ${ }^{1,2}$ Tony Van, ${ }^{3}$ Akbar K. Waljee, ${ }^{1-4}$ and Anne E. Sales ${ }^{3,5}$
}

Access to specialty care has been associated with improved survival in patients with liver disease but universal access is not always feasible. Methods of care delivery using virtual modalities including the SCAN-ECHO (Specialty Access Network-Extension of Community Healthcare Outcome) program were implemented by the Veterans Health Administration (VHA) to address this need but limited data are available on patient outcomes. We sought to evaluate the efficacy of a SCAN-ECHO visit within the context of a regional cohort of patients with liver disease in the VHA $(n=62,237)$ following implementation in the Ann Arbor SCAN-ECHO Liver Clinic from June 1, 2011, to March 31, 2015. The effect of a SCAN-ECHO visit on all-cause mortality was compared with patients with no liver clinic visit. To adjust for the differences among patients who had a SCAN-ECHO visit versus those with no visit, propensity score matching was performed on condition factors that affect the likelihood of a SCAN-ECHO visit: demographics, geographic location, liver disease diagnosis, severity, and comorbidities. During the study period, 513 patients who had a liver SCAN-ECHO visit were found within the cohort. Patients who had completed a virtual SCAN-ECHO visit were more likely younger, rural, with more significant liver disease, and evidence for cirrhosis. Propensity-adjusted mortality rates using the Cox Proportional Hazard Model showed that a SCANECHO visit was associated with a hazard ratio of 0.54 (95\% confidence interval 0.36-0.81, $P=0.003$ ) compared with no visit. Conclusion: Improved survival in patients using SCAN-ECHO suggests that this approach may be an effective method to improve access for selected patients with liver disease, particularly in rural and underserved populations where access to specialty care is limited. (Hepatology 2018;68:2317-2324).

\section{SEE EDITORIAL ON PAGE 2066}

1 The conventional approach to specialty consultation begins with a question from a primary care provider and results in an in-person visit between the specialist and the patient. Although this method has many advantages, there are also significant challenges. Most specialists cluster in urban tertiary centers, limiting access to these providers, particularly for rural patients with limited ability or means to travel. Furthermore, knowledge gaps among frontline providers, as well as other factors such as time pressures, can result in suboptimal patient care. ${ }^{(1)}$ In response to these issues, Arora et al. described an approach to specialty care using the ECHO (Extension of Community

Abbreviations: ALT, alanine aminotransferase; AUDIT-C, Alcohol Use Disorder Identification Test-C; CDW, corporate data warehouse; FIB4, Fibrosis-4; HCC, hepatocellular carcinoma; SCAN-ECHO, Specialty Access Network-Extension of Community Healthcare Outcome; VA, Veterans Affairs; VHA, Veterans Health Administration.

Received January 30, 2018; accepted April 30, 2018

Additional Supporting Information may be found at onlinelibrary.wiley.com/doi/10.1002/hep.30074/suppinfo.

Published 2018. This article is a U.S. Government work and is in the public domain in the USA.

View this article online at wileyonlinelibrary.com.

DOI 10.1002/hep.30074

Potential conflict of interest: Nothing to report. 
Healthcare Outcome) program. ${ }^{(2)}$ The ECHO program formed collaborative networks between academic specialists at the University of New Mexico and primary care providers in community-based clinics throughout the state to address inadequate access to hepatitis $\mathrm{C}$ therapy in rural communities. Akin to a virtual medicine morning report, patient cases were discussed through videoconferencing between the specialist team and primary care providers from multiple sites. Recommendations for patient care were made in real time and a didactic session was included during the conference/clinic. Continuing medical education credits were provided for all attendees for each conference, emphasizing the distance learning component of this model. ${ }^{(3)}$ In 2011, the Veterans Health Administration (VHA) adopted this program to innovate specialty care delivery and renamed the program SCAN-ECHO (Specialty Care Access NetworkExtension of Community Healthcare Outcome). The Veterans Affairs (VA) Ann Arbor Healthcare System was one of the first to implement a SCAN-ECHO program for chronic liver disease (CLD). All providers within our referral base were invited to participate in the program. We specifically queried providers to accept a SCAN-ECHO consult in place of an in-person consult in patients who did not appear to have complex medical care requiring ongoing care such as decompensated cirrhosis. However, the decision to participate was determined by the provider.

Despite wide uptake of the SCAN-ECHO program in the VHA, data are limited regarding the clinical impact of VA SCAN-ECHO, particularly on patient outcomes such as mortality. ${ }^{(2,4-10)}$ We previously showed that an in-person specialist consultation visit is associated with improved survival in liver disease patients. ${ }^{(11)}$ This study sought to extend those findings by examining whether a virtual specialty SCAN-ECHO consultation visit had a similar positive effect on survival of patients with liver disease.

\section{Materials and Methods}

\section{POPULATION}

The liver disease cohort was retrieved from the VA corporate data warehouse (CDW) and included any patients with a liver disease diagnosis (based on ICD-9 codes) in either an inpatient visit or outpatient encounter in the historical VISN (Veterans Integrated Service Network) 11, from the beginning of the Ann Arbor VA SCAN-ECHO Liver program on June 1, 2011, to March 31, 2015. The historical VISN 11 was one of 21 regions encompassing a 90,100-square-mile geographic area including Michigan, central Indiana, and northwest Ohio that was the VA Ann Arbor Healthcare System referral base during the study period. The liver disease cohort included any patient who had any one of the ICD-9 codes for liver diseases that might result in specialty care referral and were previously validated within the VHA system ${ }^{(11)}$ (Supporting Table S1). To account for late referral bias (i.e., very sick patients who may have died before being able to complete an outpatient visit), we excluded all patients who died within 6 months of their index liver disease diagnosis. This analysis, conducted as part of the operational evaluation of the Ann Arbor VA SCAN-ECHO Liver Program, was considered to be a nonresearch quality improvement under VHA Handbook 1058.05. It was conducted with the approval of the clinical leadership at the VA Ann Arbor Healthcare System.

\section{ARTICLE INFORMATION:}

From the ${ }^{1}$ Medicine Service, VA Ann Arbor Healthcare System, Ann Harbor, Michigan; ${ }^{2}$ University of Michigan Medical School, Ann Arbor, Michigan; ${ }^{3}$ VA Center for Clinical Management Research, VA Ann Arbor Healthcare System, Ann Arbor, Michigan; ${ }^{4}$ Michigan Integrated Center for Health Analytics and Medical Prediction (MiCHAMP), Ann Arbor, Michigan; ${ }^{5}$ Department of Learning Health Science, University of Michigan, Ann Arbor, Michigan.

\section{ADDRESS CORRESPONDENCE AND REPRINT REQUESTS TO:}

Grace L. Su, M.D.

VA Ann Arbor Healthcare System

2215 Fuller Road
Ann Arbor, MI 48105

E-mail: gsu@umich.edu

Tel: +1-734-845-5865 


\section{PRIMARY OUTCOME}

The primary outcome was all-cause mortality as determined by linking the patient data files with the Vital Status Files in the CDW. Deaths were recorded from the beginning of the study period (June 1,2011) until March 31, 2015.

\section{VETERANS AFFAIRS SCAN-ECHO VISIT GROUP}

The intervention of primary interest was a VA Liver SCAN-ECHO consultation visit. Since the inception of the Ann Arbor VA SCAN-ECHO clinic in June 2011, the patients who were discussed at these clinics were tracked prospectively. A total of 520 unique patients had a VA SCAN-ECHO visit during the study period. Of these, 513 were found within the cohort that was extracted from the CDW. Seven were excluded because the only ICD 9 code they had was for abnormal imaging of the gastrointestinal tract. This code was not included in our liver disease patient cohort, as this code is not specific for liver disease (Supporting Fig. S1).

\section{NO VISIT GROUP}

Within the population of patients with liver disease, we defined patients as having no visit if they were not in the VA SCAN-ECHO cohort and did not receive a traditional in-person hepatology consultation visit during the study period, as indicated by the presence of codes in the electronic record indicating a completed liver clinic visit.

\section{PREDICTORS OF A SCAN-ECHO VISIT}

Predictors of a visit were defined as follows: demographic predictors were age at diagnosis, gender, and race/ethnicity. Urban, rural, and highly rural patient residence was determined based on VA Planning Systems Support Group geocoding. ${ }^{(12)}$ Liver disease diagnoses (hepatitis B and C), cirrhosis and cirrhosis comorbidities, including ascites, spontaneous bacterial peritonitis, hepatorenal syndrome, variceal bleed, hepatocellular carcinoma, hepatic encephalopathy and thrombocytopenia, were identified by ICD 9 codes. Cirrhosis complications were defined by the presence of codes for variceal bleeding, ascites, and hepatic encephalopathy. The alanine aminotransferase (ALT) value used was the highest ALT level within 365 days, but prior to diagnosis, to account for triggers for consultation. The bilirubin level used was the value closest to diagnosis date and within 180 days prior to diagnosis to account for severity of liver disease. Modified Elixhauser comorbidity scores were calculated using diagnosis codes that were present 365 days prior to the first liver disease diagnosis and excluded liver disease and alcoholism. ${ }^{(13)}$ The presence of a procedure (i.e., abdominal computed tomography [CT], magnetic resonance imaging [MRI], abdominal ultrasound, esophagoduodenoscopy, liver biopsy, and paracentesis) was defined by the presence of a current procedural terminology code for the procedure within the 1 year prior to enrollment into the cohort.

\section{STATISTICAL ANALYSIS}

Baseline covariates were defined within 1 year of the index date, defined as the first appearance of any of the liver disease diagnosis codes. We conducted two separate analyses: (1) a descriptive analysis of veterans in an unmatched cohort with a VA SCAN-ECHO visit compared with those with no visit, and (2) a survival analysis using a propensity score-matched cohort to evaluate mortality among veterans with a VA SCAN-ECHO visit compared with those with no visit. Analysis of variance with Tukey's adjustment for multiple group comparisons was used for bivariate analyses of categorical and continuous predictors of access. Multivariable logistic regression was performed to determine independent predictors of a SCAN-ECHO visit. Survival analysis was performed to analyze the time from index date to death or end of follow-up (April 1, 2015). All relevant variables were included in the final model regardless of their statistical significance in bivariate testing.

To determine whether a SCAN-ECHO visit was independently associated with mortality, the propensity score model was developed, adjusting for the likelihood of a SCAN-ECHO visit using a binary logistic regression on the baseline covariates. The SCAN-ECHO patients were matched 1:1 to patients with no visit, taking the closest or best available match without replacement. The quality of the matching was assessed using the absolute standardized mean difference as a balance measure. Survival differences were computed using the Cox Proportional Hazard models and Kaplan-Meier survival curves. Additional sensitivity analyses were performed after dividing the cohorts into two groups based 
on a Fibrosis-4 (FIB-4) score as a surrogate for presence of advanced liver disease/cirrhosis. ${ }^{(14)}$ A cutoff of greater than 3.25 was used to identify patients with a high probability of advanced liver disease. ${ }^{(15)}$ All statistical analyses were performed using the statistical language $\mathrm{R}$, using the packages MatchIt (for propensity matching) and Survival (for Cox proportional hazard modeling).

\section{Results}

\section{DEMOGRAPHICS AND CLINICAL CHARACTERISTICS}

During the study period, 513 eligible patients had a VA SCAN-ECHO visit and 62,237 had no visit within a cohort of 67,314 patients with a liver disease diagnosis (Table 1, Supporting Fig. S1). A total of 4,564 patients had a traditional in-person liver clinic visit and were excluded from the no visit group. In comparing the characteristics of patients who received a SCAN-ECHO consultation versus no visit, the patients in the SCAN-ECHO visit group were more likely rural or highly rural. This reflects the particular affinity for providers and patients to use a virtual modality when patients live far from a specialty site. Patients with a well-characterized liver diagnosis such as hepatitis $\mathrm{B}$, hepatitis $\mathrm{C}$, cirrhosis, and cirrhosis complications were more likely to have had a SCAN-ECHO visit. This is consistent with prior patterns seen, in which patients who had

TABLE 1. Characteristics of the Unmatched Cohorts

\begin{tabular}{|c|c|c|c|}
\hline & VA SCAN-ECHO & No visit & Significance \\
\hline $\mathrm{N}$ & 513 & 62,237 & - \\
\hline Age (SD) & $56.4(11.5)$ & $58.0(15.2)$ & NS \\
\hline Male gender (\%) & $485(94.5)$ & $58,698(94.3)$ & NS \\
\hline Race (\%) & & & NS \\
\hline White & $370(72.1)$ & $45,840(73.7)$ & \\
\hline Nonwhite & $104(20.3)$ & $12,860(20.7)$ & \\
\hline Unknown & $39(7.6)$ & $3537(5.7)$ & \\
\hline Geographic (\%) & & & $*$ \\
\hline Urban & $240(46.8)$ & $38,262(61.5)$ & \\
\hline Rural & $262(51.1)$ & $23,408(37.6)$ & \\
\hline Highly rural & $11(2.1)$ & $567(0.9)$ & \\
\hline Hepatitis B (\%) & $14(2.7)$ & $434(0.7)$ & $*$ \\
\hline Hepatitis C (\%) & $225(43.9)$ & $7438(12.1)$ & $*$ \\
\hline Cirrhosis (\%) & $85(16.6)$ & $2032(3.3)$ & $*$ \\
\hline Cirrhosis complication (\%) & $22(4.3)$ & $774(1.2)$ & $*$ \\
\hline Ascites (\%) & $13(2.5)$ & $120(2.6)$ & $*$ \\
\hline Hepatorenal syndrome (\%) & $1(0.2)$ & $40(0.1)$ & NS \\
\hline Spontaneous bacterial peritonitis (\%) & $0(0.0)$ & $59(0.1)$ & NS \\
\hline Variceal bleed (\%) & $8(1.6)$ & $145(0.2)$ & $*$ \\
\hline Hepatic encephalopathy (\%) & $6(1.2)$ & $267(0.4)$ & $*$ \\
\hline Hepatocellular carcinoma (\%) & $9(1.8)$ & $332(0.5)$ & $*$ \\
\hline Thrombocytopenia (\%) & $50(9.7)$ & $1507(2.4)$ & $*$ \\
\hline $\operatorname{ALT}(\mathrm{U} / \mathrm{L})(\mathrm{SD})$ & $89.5(93.6)$ & $50.5(98.4)$ & $*$ \\
\hline Bilirubin (mg/dL) (SD) & $0.76(0.98)$ & $0.65(0.89)$ & $*$ \\
\hline Modified Elixhauser comorbidity (SD) & $0.70(0.93)$ & $0.62(0.91)$ & $*$ \\
\hline Abdominal CT (\%) & $110(21.4)$ & $10,099(16.2)$ & $*$ \\
\hline Abdominal MRI (\%) & $17(3.3)$ & $1373(2.2)$ & NS \\
\hline Abdominal ultrasound (\%) & $288(56.1)$ & $9403(15.1)$ & $*$ \\
\hline Esophagoduodenoscopy (\%) & $61(11.9)$ & $3343(5.4)$ & $*$ \\
\hline Liver biopsy (\%) & $9(1.8)$ & $288(0.5)$ & $*$ \\
\hline Paracentesis (\%) & $1(0.2)$ & $252(0.4)$ & NS \\
\hline
\end{tabular}

${ }^{*} P$ value $<0.05$.

Abbreviation: NS, nonsignificant. 
these diagnoses were more likely to have a specialty consultation leading to a visit. ${ }^{(11)}$ Similarly, patients with indicators suggestive of more severe liver disease such as elevated ALT and bilirubin were more likely to have had a SCAN-ECHO visit than no visit. The SCAN-ECHO visit group also had more imaging or endoscopic procedures, which is consistent with our prior reports that patients who had specialty consultation were more likely to have more imaging studies and procedures. ${ }^{(11)}$ In the multivariable analysis, the likelihood of having a SCAN-ECHO visit was increased in patients who were younger, who lived in a rural location, who had an ultrasound performed, or who had a diagnosis of cirrhosis complication, hepatitis $\mathrm{C}$, or thrombocytopenia.

\section{IMPROVED SURVIVAL AMONG PATIENTS WITH LIVER DISEASE DIAGNOSIS AND HAVING HAD A SCAN-ECHO VISIT}

The effect of a SCAN-ECHO visit on all-cause mortality was compared with patients with no visit. To adjust for the differences among patients who had a SCAN-ECHO visit versus no visit, propensity score matching was performed. Propensity scores were developed on factors that affect the likelihood of a SCAN-ECHO visit (Table 1). The balance of matching is illustrated in Fig. 1 (SCAN-ECHO versus no visit). In the matched cohorts, patients who had a SCAN-ECHO visit had significantly improved survival as compared with those who had no visit (Fig. 2). Compared with having no visit, matched patients who had consultation through the SCAN-ECHO program were associated with decreased risk of death (hazard ratio $0.54,95 \%$ confidence interval 0.36-0.81, $P=0.003$ ), with a $46 \%$ decreased likelihood of dying compared with the matched patients who had no visit during the follow-up period. Although our primary comparison was between those patients who had a SCAN-ECHO visit versus those who had no visit, we also examined whether specialty care delivery using SCAN-ECHO might result in a different survival benefit than those with an in-person visit. We found that using the same propensity score matching methods that matched cohorts of patients with SCAN$\mathrm{ECHO}$ visits and traditional in-person liver visits had similar survival (Supporting Fig. S2).

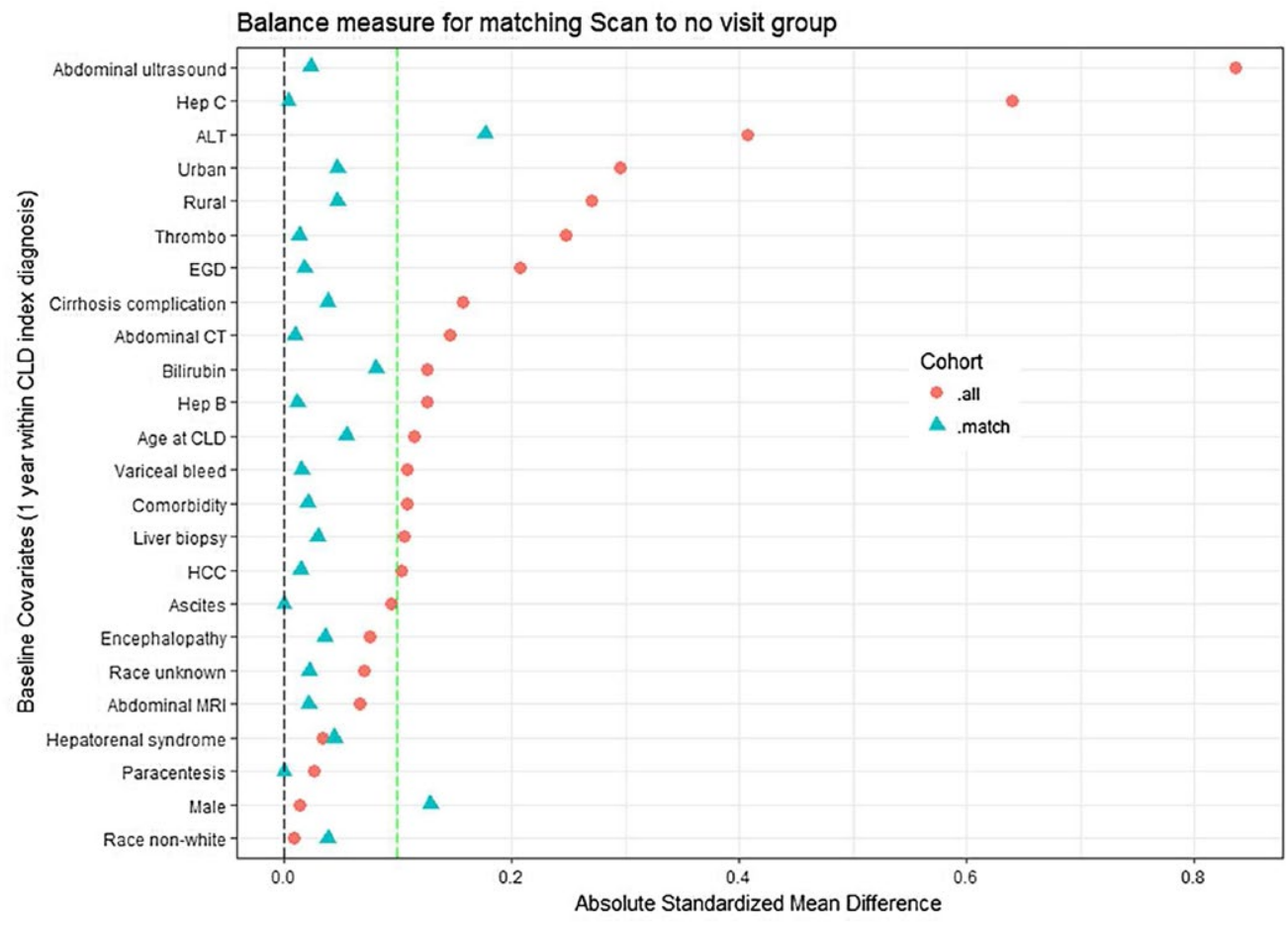

FIG. 1. Absolute standardized mean difference of the baseline covariates between patients with a SCAN-ECHO visit versus no visit. 


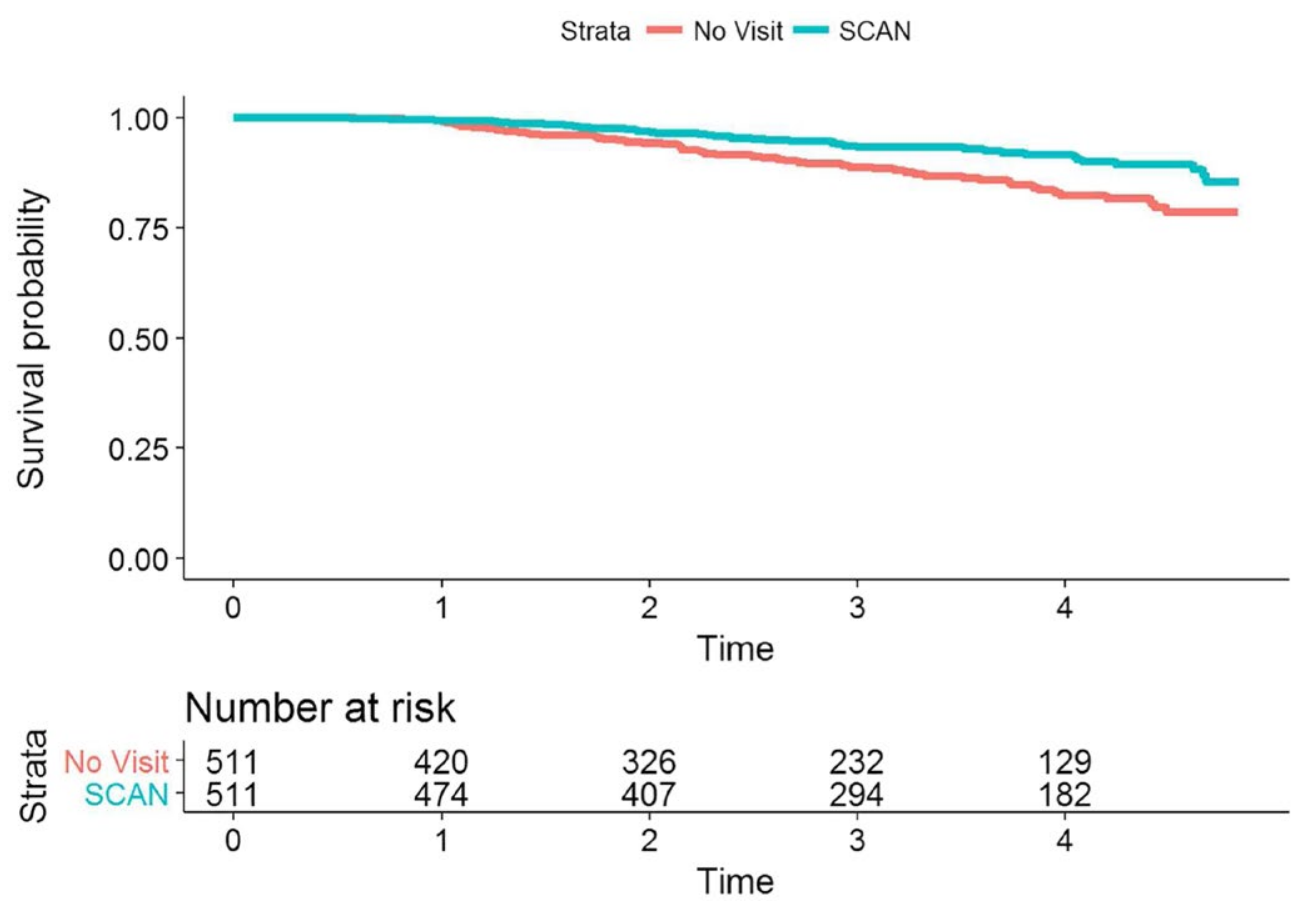

FIG. 2. Kaplan-Meier survival curve between those with a SCAN-ECHO versus propensity-matched cohort of patients with no visit.

To examine whether the reduction in mortality was only seen in patients with advanced liver disease or all patients, we divided the matched cohorts by their FIB-4 score. In the propensity-matched cohorts, a SCAN-ECHO visit was associated with reduced risk of death in patients with and without advanced fibrosis. A SCAN-ECHO visit was associated with a decreased risk of death compared with those with no visit, with a hazard ratio of 0.40 in the cohorts of patients whose FIB-4 was greater than $3.25(n=260$, $P=0.0004)$ and 0.42 in patients whose FIB-4 score was less than $3.25(n=676, P=0.03)$. The analysis was performed only on patients who had complete data for FIB-4.

To examine whether the matched cohorts had differences in alcohol intake, we examined the Alcohol Use Disorder Identification Test-C (AUDIT-C) scores in the propensity-matched cohorts. The AUDIT-C scores are widely implemented within the VHA and are mandated as a performance measure. ${ }^{(16)}$ This simple, three-question questionnaire has been validated within and outside the VHA for identifying patients with alcohol use disorder. ${ }^{(17,18)}$ We found in our matched cohorts that the AUDIT-C scores were similar between those with a SCAN-ECHO visit and those with no visit $(1.96 \pm 2.93$ versus $1.93 \pm 2.73$, $P>0.05)$.

Seeking potential mechanisms for improved survival, we examined the relative frequency of hepatocellular carcinoma (HCC) surveillance (defined as an abdominal imaging study at least once a year) and variceal surveillance (defined as an esophagogastroduodenoscopy (EGD) at least every 3 years) in the unmatched cohort of patients with FIB-4 scores greater than 3.25. We found that those with a SCAN$\mathrm{ECHO}$ visit had a higher rate of $\mathrm{HCC}$ (42\% versus $25 \%)$ and variceal surveillance (25\% versus $15 \%)$ compared with those with no visit.

\section{Discussion}

Novel strategies are needed to provide specialty care to patients with complex liver disease whose access is limited by geographic distance from referral centers. We evaluated the SCAN-ECHO program, a virtual form of electronic consultation, which combines real-time consultation with didactic learning for front-line providers. In this large regional cohort of patients with liver disease, we found that patients who 
received consultation through the SCAN-ECHO program had improvements in several measures of care quality and higher survival rates than patients who had no specialty care visit. These data extend the findings of prior studies in which we and others have found a benefit of access to traditional, in-person specialty care consultations in patients with liver disease. ${ }^{(11,19-21)}$ Previously, the $\mathrm{ECHO}^{(2,22-24)}$ and other more traditional (involving direct patient to provider contact $)^{(25,26)}$ telemedicine programs have been reported, with some showing effectiveness for treating hepatitis $\mathrm{C}$ both within and outside of the VHA with equal outcomes. In this study, we extend the impact of SCAN-ECHO beyond hepatitis $\mathrm{C}$ therapy to show that this intervention is associated with improved survival for patients with all liver diseases.

Beyond the novelty of the intervention, we found several strengths in these analyses. First, we found benefit even after applying propensity matching using broad demographic and clinical characteristics to adjust for effect estimates. Second, we demonstrated clinically significant improvements in screening procedures and enhanced survival benefit for patients with elevated FIB-4 scores (indicating cirrhosis), establishing a mechanism for the observations. Third, based on the Kaplan-Meier analysis, the improved outcomes that are specific to the SCAN-ECHO program emerge after 1 year, suggesting both equal matching at baseline and plausible effects based on the types of intervention differences that may have resulted from specialist consultation (i.e., varices or HCC screening).

These data must be interpreted in the context of the study design. First, the consultations addressed many issues for patients with diverse liver diseases. Accordingly, the underlying mechanism for generalized improvement in mortality is not clear from these data. We present analyses, however, that demonstrate beneficial effects for patients at the highest risk of death, namely those with possible cirrhosis (FIB-4 > 3.25). Specifically, we show that SCAN$\mathrm{ECHO}$ is associated with improved process measures such as screening for liver cancer and varices that could be linked to improved survival. Furthermore, although patients were not randomized to receive either form of consultation, we adjusted for confounders using propensity-score matching. Although every attempt was made to match patient characteristics between control and cases, certain features such as patient socioeconomic status were not completely available. There is always the concern that we did not account for all patient differences or eliminate confounding by indication. Second, our propensity matching only accounted for patient factors. It is possible that the difference may be due to provider-level factors that are not accounted for here. All providers within our referral base were offered the option to participate in the program. However, as all consultations were voluntary, the choice to accept the use of the SCAN$\mathrm{ECHO}$ visit on the part of the front-line provider may reflect providers with baseline-elevated knowledge or interest in practice improvement. We plan to study this as a possible mechanism for improved survival. In addition to intrinsic provider differences, the ECHO program may allow for more effective dissemination of patient-specific information or improved knowledge base. A prior study on the improvement of knowledge base using provider surveys has shown surprising dissemination of liver knowledge. ${ }^{(27)}$ Third, these data reflect the VA experience, which is a fully integrated healthcare system with a unique population. Both factors may constrain the generalizability of these findings to other settings. Regulatory issues such as state-specific licensing and legal issues such as malpractice may be limitations in non-VA settings.

In a highly integrated healthcare system, an approach such as the SCAN-ECHO program may be an effective, scalable method to improve access for selected patients with liver disease, particularly in rural and underserved populations where access to specialty care is limited.

\section{REFERENCES}

1) Beste LA, Harp BK, Blais RK, Evans GA, Zickmund SL. Primary care providers report challenges to cirrhosis management and specialty care coordination. Dig Dis Sci 2015;60:2628-2635.

2) Arora S, Thornton K, Murata G, Deming P, Kalishman S, Dion $\mathrm{D}$, et al. Outcomes of treatment for hepatitis $\mathrm{C}$ virus infection by primary care providers. N Engl J Med 2011;364:2199-2207.

3) Arora S, Kalishman S, Thornton K, Dion D, Murata G, Deming $\mathrm{P}$, et al. Expanding access to hepatitis $\mathrm{C}$ virus treatmentExtension for Community Healthcare Outcomes (ECHO) project: disruptive innovation in specialty care. Hepatology 2010;52:1124-1133.

4) Kirsh S, Su GL, Sales A, Jain R. Access to outpatient specialty care: solutions from an integrated health care system. Am J Med Qual 2015;30:88-90.

5) Stevenson L, Ball S, Haverhals LM, Aron DC, Lowery J. Evaluation of a national telemedicine initiative in the Veterans Health Administration: factors associated with successful implementation. J Telemed Telecare 2018;24:168-178. 
6) Watts SA, Roush L, Julius M, Sood A. Improved glycemic control in veterans with poorly controlled diabetes mellitus using a Specialty Care Access Network-Extension for Community Healthcare Outcomes model at primary care clinics. J Telemed Telecare 2016;22:221-224.

7) Glass LM, Waljee AK, McCurdy H, Su GL, Sales A. Specialty Care Access Network-Extension of Community Healthcare Outcomes model program for liver disease improves specialty care access. Dig Dis Sci 2017;62:3344-3349.

8) Zhou C, Crawford A, Serhal E, Kurdyak P, Sockalingam S. The impact of project ECHO on participant and patient outcomes: a systematic review. Acad Med 2016;91:1439-1461.

9) Carey EP, Frank JW, Kerns RD, Ho PM, Kirsh SR. Implementation of telementoring for pain management in Veterans Health Administration: spatial analysis. J Rehabil Res Dev 2016;53:147-156.

10) Sayre GG, Haverhals LM, Ball S, Stevenson L, Battaglia C, Aron DC, et al. Adopting SCAN-ECHO: the providers' experiences. Healthc (Amst) 2017;5:29-33.

11) Mellinger JL, Moser S, Welsh DE, Yosef MT, Van T, McCurdy $\mathrm{H}$, et al. Access to subspecialty care and survival among patients with liver disease. Am J Gastroenterol 2016;111:838-844.

12) West AN, Lee RE, Shambaugh-Miller MD, Bair BD, Mueller KJ, Lilly RS, et al. Defining "rural" for veterans' health care planning. J Rural Health 2010;26:301-309.

13) Elixhauser A, Steiner C, Harris DR, Coffey RM. Comorbidity measures for use with administrative data. Med Care 1998;36:8-27.

14) Tapper EB, Lok AS. Use of liver imaging and biopsy in clinical practice. N Engl J Med 2017;377:2296-2297.

15) Vallet-Pichard A, Mallet V, Nalpas B, Verkarre V, Nalpas A, Dhalluin-Venier $V$, et al. FIB-4: an inexpensive and accurate marker of fibrosis in $\mathrm{HCV}$ infection. comparison with liver biopsy and fibrotest. Hepatology 2007;46:32-36.

16) Bradley KA, Williams EC, Achtmeyer CE, Volpp B, Collins BJ, Kivlahan DR. Implementation of evidence-based alcohol screening in the Veterans Health Administration. Am J Manag Care 2006;12:597-606.

17) Bush K, Kivlahan DR, McDonell MB, Fihn SD, Bradley KA. The AUDIT alcohol consumption questions (AUDIT-C): an effective brief screening test for problem drinking. Ambulatory Care Quality Improvement Project (ACQUIP). Alcohol Use Disorders Identification Test. Arch Intern Med 1998;158:1789-1795.
18) Bradley KA, DeBenedetti AF, Volk RJ, Williams EC, Frank D, Kivlahan DR. AUDIT-C as a brief screen for alcohol misuse in primary care. Alcohol Clin Exp Res 2007;31:1208-1217.

19) Davila JA, Kramer JR, Duan Z, Richardson PA, Tyson GL, Sada YH, et al. Referral and receipt of treatment for hepatocellular carcinoma in United States veterans: effect of patient and nonpatient factors. Hepatology 2013;57:1858-1868.

20) Kanwal F, Kramer JR, Buchanan P, Asch SM, Assioun Y, Bacon BR, et al. The quality of care provided to patients with cirrhosis and ascites in the Department of Veterans Affairs. Gastroenterology 2012;143:70-77.

21) Ko CW, Kelley K, Meyer KE. Physician specialty and the outcomes and cost of admissions for end-stage liver disease. Am J Gastroenterol 2001;96:3411-3418.

22) Beste LA, Glorioso TJ, Ho PM, Au DH, Kirsh SR, ToddStenberg J, et al. Telemedicine specialty support promotes hepatitis $\mathrm{C}$ treatment by primary care providers in the Department of Veterans Affairs. Am J Med 2017;130:432-438.

23) Serper M, Volk ML. Current and future applications of telemedicine to optimize the delivery of care in chronic liver disease. Clin Gastroenterol Hepatol 2018;16:157-161.

24) Rossaro L, Torruellas C, Dhaliwal S, Botros J, Clark G, Li CS, et al. Clinical outcomes of hepatitis $\mathrm{C}$ treated with pegylated interferon and ribavirin via telemedicine consultation in Northern California. Dig Dis Sci 2013;58:3620-3625.

25) Cooper CL, Hatashita H, Corsi DJ, Parmar P, Corrin R, Garber G. Direct-acting antiviral therapy outcomes in Canadian chronic hepatitis C telemedicine patients. Ann Hepatol 2017;16: 874-880.

26) You A, Kawamoto J, Smith JP. A pharmacist-managed telemedicine clinic for hepatitis $\mathrm{C}$ care: a descriptive analysis. J Telemed Telecare 2014;20:99-101.

27) Salgia RJ, Mullan PB, McCurdy H, Sales A, Moseley RH, $\mathrm{Su}$ GL. The educational impact of the Specialty Care Access Network-Extension of Community Healthcare Outcomes program. Telemed J E Health 2014;20:1004-1008.

\section{Supporting Information}

Additional Supporting Information may be found at onlinelibrary.wiley.com/doi/10.1002/hep.30074/suppinfo. 